\title{
The effects of sleep and light at night on melatonin in adolescents
}

\author{
Caleb Hersh, ${ }^{1}$ Julia Sisti, ${ }^{2}$ Vincent Richiutti, ${ }^{3}$ Eva Schernhammer ${ }^{2,4,5}$
}

\begin{abstract}
${ }^{1}$ The College of Brown University, Brown University, Providence, RI, ${ }^{2}$ Department of Epidemiology, Harvard T.H. Chan School of Public Health, Boston, MA, ${ }^{3}$ UC Health University Hospital Clinical Laboratories, University of Cincinnati College of Medicine, Cincinnati, $\mathrm{OH},{ }^{4}$ Channing Division of Network Medicine, Department of Medicine, Brigham and Women's Hospital and Harvard Medical School, Boston, MA, USA, ${ }^{5}$ Applied Cancer Research-Institution for Translational Research Vienna (ACR-ITR VIEnna), Vienna, Austria
\end{abstract}

\begin{abstract}
OBJECTIVE: The circadian hormone melatonin has wide-reaching effects on human physiology. In adolescents, the impact of nighttime light exposure and other modifiable behavioral factors on melatonin levels is poorly understood. DESIGN: We cross-sectionally examined the influence of nighttime behaviors on melatonin levels in 100 adolescents (average age: 15.7; 55 female, 45 male), who completed a self-administered questionnaire and provided a first morning urine sample to assay for urinary 6-sulfatoxymelatonin (aMT6s) levels. We used mixed-effects regression models to test for differences in aMT6s levels by categories of covariates. RESULTS: Self-reported sleep duration, ambient light levels during sleep, and use of electronics after turning off lights did not significantly predict aMT6s levels. Compared to those who reported weekend bedtimes before $11 \mathrm{pm}$, urinary aMT6s levels were significantly lower among participants reporting weekend bedtimes after midnight (52.5 vs. $38.0 \mathrm{ng} / \mathrm{mg}$ creatinine, $\left.\boldsymbol{P}_{\text {trend }}=\mathbf{0 . 0 0 7}\right)$. Sleep interruption also appeared to be significantly associated with lower urinary aMT6s levels, but only if lights were turned on during sleep interruption (43.0 $\mathrm{ng} / \mathrm{mg}$ creatinine for participants with sleep interruption but not turning lights on, vs. 24.6 $\mathbf{n g} / \mathbf{m g}$ creatinine for participants reporting that they turned on the light when their sleep was interrupted $\boldsymbol{P}_{\text {difference }}=0.032$ ). CONCLUSIONS: Our study suggests that self-reported sleeprelated behaviors have little to no effect on adolescent circadian systems, though larger studies are needed to confirm our findings.
\end{abstract}

Key words: Adolescents, Light at night, 6-sulfatoxymelatonin, Sleep duration

Address for correspondence:

Julia Sisti, Harvard T.H. Chan School of Public Health, 677 Huntington Avenue, Boston, MA 02115, USA,

Tel.: (201) 694-2077, E-mail: jss235@mail.harvard.edu

Received 09-08-2014, Accepted 22-05-2015

\section{INTRODUCTION}

National-level data indicate that sleep problems are endemic among US teenagers. ${ }^{1}$ The circadian system undergoes rapid changes in puberty that may predispose adolescents to fall asleep and wake up 
at later times than adults., ${ }^{2,3}$ Often, this developing physiology conflicts with early school start times: in a national survey, nearly half of adolescent respondents reported that they slept less than the recommended 8 hours on weeknights. ${ }^{1}$ Sleep deprivation is associated with a number of adverse outcomes in youths, including impairments in mood and behavior, ${ }^{4}$ poor academic performance, ${ }^{5}$ and possibly obesity. ${ }^{6}$ In adults, disruption of the circadian sleep cycle has been associated with an increased risk of cancer. ${ }^{7}$

While some evidence suggests that delaying school start times even by a small amount may decrease fatigue and improve mood, ${ }^{8}$ implementing changes to school schedules is not generally a feasible option for most school districts. As a result, more research is needed to quantify the impact of modifiable factors - such as nighttime light exposure - on adolescent health.

The effects of sleep on health may be mediated through the circadian hormone melatonin, which has wide-reaching effects on human physiology and is an emerging biomarker for adult chronic diseases. Melatonin secretion in humans exhibits diurnal variation: levels are lowest during the day and peak overnight during sleep. ${ }^{9}$ Melatonin release from the pineal gland may also be suppressed by exogenous factors, particularly natural and artificial light. ${ }^{10}$ In studies of adults, significant inverse associations between exposure to light at night - often resulting from nightshift work - and melatonin levels have been documented. ${ }^{11}$ However, the relationships between similar exposures and melatonin levels in younger populations have not been widely studied.

In the present analysis, we cross-sectionally examined associations between nighttime behaviors and urinary markers of melatonin levels in adolescents. All participants provided self-reported information about sleep-related factors, while a first-morning urine sample was assayed to quantify melatonin levels on a mid-week night during the school year.

\section{SUBJECTS \& METHODS}

\section{Study population and procedures}

Between the months of April and May 2011, students in grades 9-12 at a suburban New York high school were recruited for participation via flyers and in-classroom presentations by one of the investigators (C.H). All students willing to answer a short questionnaire and provide a first-morning urine sample on a designated study date were eligible for inclusion. Written consent was obtained from all participants and from the parent or guardian of each participant. All procedures were conducted with approval of the Ossining High School Institutional Review Board.

\section{Instrumentation}

Participants were given two days in late May 2011 to complete a paper questionnaire aimed at assessing nighttime habits, light exposure, and sleep duration. In addition to assessing demographic and lifestyle characteristics, questionnaire items queried: 1) Time at which participants turned off the light to go to sleep and time at which they woke up on each day of a usual week; 2) Number of hours usually spent looking at an electronic device (e.g. laptop or desktop computer, television) after lights are turned off to go to sleep; 3 ) Use of sleep aids and type; 4) Whether a light or television was turned on in, or near, the bedroom while sleeping; 5) Average number of times per night, if any, that their sleep was interrupted; 6) If sleep was interrupted, participants were asked to indicate whether they turned a light on, and intensity (bright or dim) and duration $(<5$, 5-10, or $\geq 10$ minutes) of light exposure; 7) Average sleep duration (in hours) for weekdays and weekend nights, separately; 8) Level of ambient light present in the bedroom during sleep on weekdays, weekends, and night prior to urine collection.

Ambient light levels were classified according to the numerical scale established by Davis et $\mathrm{a}^{12}: 1$ ) Subject wears a mask to keep out the light; 2) Subject cannot see his/her hand in front of his/her face; 3) Subject can see to the end of the bed; 4) Subject can see across the room; 5) Subject can barely read; and 6) Subject can read comfortably.

Time spent in bed on weekends and weekdays was calculated as the difference between the hour at which participants reported getting into bed with the lights off and the hour at which they reported waking. Participants were also asked to provide the location of their current residence, duration of residence at that address, and whether artificial outdoor light was visible from their bedroom window. 
Between June and July 2012, additional information about participants' chronotype was collected via email. Respondents answered a single question from the Horne-Ostberg Morningness-Eveningness Questionnaire ${ }^{13}$ indicating whether they most closely identified as: 1) A definite "morning” type; 2) Rather more of a "morning" type than an "evening" type; 3 ) Rather more of an "evening" type than a "morning" type; or 4) A definite "evening" type. This item has previously been shown to correspond well to the overall questionnaire score. ${ }^{14}$

\section{Urine collection}

On May 25, 2011, all participants collected firstmorning urine samples in their homes using $4.5 \mathrm{~mL}$ tubes provided by the investigators. All samples were collected when participants arrived at school, between approximately 7:30 and 8:00 am, and were immediately placed on ice and transported within 6 hours to the Specialty Assay Research Core Lab (Boston, MA). Eleven random urine samples were split as blinded quality control and quality assurance for the laboratory analysis.

\section{Laboratory methods}

Urinary melatonin (6-sulfatoxymelatonin, aMT6s) measurements were assayed using commercially available ELISA kits (ALPCO, Windham, NH). The Bühlmann 6-SMT ELISA is an immunoassay using an antibody-capture technique with a lower detection limit of $0.8 \mathrm{ng} / \mathrm{ml}$ for aMT6s. All aMT6s assays are creatinine-standardized to account for differences arising from variations in urine concentrations. Urine creatinine was measured using the COBAS Integra 400 (Roche Diagnostics, Indianapolis, IN). To assess reproducibility of the laboratory methods, masked split specimens included within each batch $(10 \%$ of the total samples) were used to calculate the coefficient of variation within batches; for urinary aMT6s the average within-batch $\mathrm{CV}$ among the samples was $3.3 \%$ and for creatinine, $1.4 \%$.

\section{Statistical methods}

No study participants were missing aMT6s levels and none of the aMT6s values were identified as outliers based on the generalized extreme studentized deviate many-outlier detection approach, ${ }^{15}$ leaving a total of 100 students available for our analyses.

All statistical analyses were performed with SAS software (SAS Institute, Cary, NC). Fisher's exact tests were used to examine univariate relationships between chronotype and reported sleep habits. Continuous variables in Table 1 are presented as means (standard deviation, SD). To test for differences in aMT6s levels by categories of covariates, we used mixed-effects regression models to obtain geometric mean aMT6s levels and $95 \%$ confidence intervals $(95 \% \mathrm{CI})$. Main models were adjusted for age (years, continuous),

Table 1. Characteristics of 100 study participants, overall and by gender*

\begin{tabular}{lccc}
\hline Characteristic & Overall & Females & Males \\
\hline $\mathrm{N}(\%)$ & $100(100.0)$ & $55(55.6)$ & $44(44.4)$ \\
$\mathrm{Age}$, years & $15.7(1.2)$ & $15.8(1.3)$ & $15.5(1.0)$ \\
$\mathrm{BMI}, \mathrm{kg} / \mathrm{m}^{2}$ & $21.6(3.5)$ & $22.0(4.0)$ & $21.1(2.8)$ \\
Melatonin level, $\mathrm{ng} / \mathrm{mL}$ & 72.3 & 71.4 & 71.2 \\
Creatinine level, $\mathrm{mg} / \mathrm{dL}$ & 158.6 & 152.5 & 165.0 \\
Age at first menstrual period, median (range) & $\mathrm{N} / \mathrm{A}$ & $12(9-14)$ & $\mathrm{N} / \mathrm{A}$ \\
Caucasian (\%) & $67(67.8)$ & $33(61.1)$ & $33(75.0)$ \\
Reported tobacco use & $2(2.0)$ & $0(0.0)$ & $2(4.8)$ \\
Reported alcohol use in past 30 days & $18(18.6)$ & $11(20.0)$ & $7(17.9)$ \\
Current OC Use & $\mathrm{N} / \mathrm{A}$ & $5(9.3)$ & $\mathrm{N} / \mathrm{A}$ \\
Exercise 4 or more hours/week & $67(67.1)$ & $35(64.8)$ & $32(76.2)$ \\
Use of sleep aid in past 3 months & $8(8.0)$ & $6(10.9)$ & $2(4.6)$ \\
\hline
\end{tabular}

*One individual did not provide data on gender but was retained in all analyses not stratified by sex; ${ }^{\dagger}$ Females only. 
sex, body mass index $\left(\mathrm{kg} / \mathrm{m}^{2}\right.$, continuous), alcohol consumption (any vs. none), physical exercise $(<4$ hours per week vs. $4+$ hours per week), and ethnicity (white/Caucasian vs. other). Further adjustment for sleep duration did not materially change results and this covariate was not retained in final models. When the underlying variable was continuous, such as age or BMI, $P$-values are reported for the linear trend test. For categorical variables, we estimated $P$ for trend by modeling categories ordinally or by modeling the medians of the categories continuously. In secondary analyses, we also calculated $P$ for differences between extreme categories. We present them only for light exposure during sleep interruption (levels among those whose sleep was never interrupted vs. levels among those who reported turning a light on when their sleep was interrupted), as this was the only variable where we observed significant differences. All $P$-values were based on two-sided tests and were considered statistically significant if $\leq 0.05$. Complete case approach was used for all analyses.

In sensitivity analyses, we excluded smokers, those who reported using sleep aids, and female participants reporting the use of oral contraceptives. Because their exclusion did not alter our findings, we retained these individuals in our main analyses. In secondary analyses exploring the association between light outside the bedroom window and urinary melatonin levels, we excluded the two students who reported having lived at their current residence for 8 months or fewer.

\section{RESULTS}

\section{Study population}

Characteristics of the study participants are shown in Table 1. Fifty-five participants (55\%) were female and $44(44 \%)$ were male. One participant did not report sex; this individual was retained in all analyses not stratified by sex. Ages of participants ranged from 14 to 18 years, with a mean age of $15.7(\mathrm{SD}=1.2)$. BMI ranged from 15.4 to $37.1 \mathrm{~kg} / \mathrm{m}^{2}$, with a mean of $21.6(\mathrm{SD}=3.5)$. Students predominantly identified as Caucasian (67.8\%).

\section{Sleep habits}

Both self-reported sleep duration and calculated time spent in bed with the lights off were longer on the weekends than the weekdays. On weekdays, average self-reported sleep duration was 6.9 hours $(\mathrm{SD}=1.03)$, while average time spent in bed was 7.7 hours $(\mathrm{SD}=0.86)$. The Spearman correlation between these two measures was $0.45(P<0.001)$. During the weekend, average self-reported sleep duration was 8.6 hours $(\mathrm{SD}=1.42)$ and average time spent in bed was 9.2 hours $(\mathrm{SD}=1.4)$. These two measures were also significantly correlated with a Spearman correlation coefficient of $0.43(P<0.001)$.

The finding that time spent in bed was longer than the self-reported sleep duration indicated that participants had at least some amount of time between turning off the lights and falling asleep. On weekdays, $21 \%$ of participants reported using electronics for more than one hour after turning the lights out and before going to sleep, compared to $49 \%$ on the weekends. Other sources of ambient light at night reported by participants included light outside their bedroom window at night (45\%) and $22.2 \%$ of participants reported that there was a TV or light on inside or near their bedroom while they slept. Asked to rate the brightness level in their rooms during the weekday and weekend nights, the largest proportion of students reported that they "could see to the end of the bed" (38.0\% and 38.4\%, respectively).

Twenty-five (25) participants (25.3\%) reported having their sleep interrupted up to 4 times a night. Among this subset, 4 participants - all females - reported turning on a light when their sleep got interrupted. Of those who reported turning on a light when sleep was interrupted, one reported that the light was dim, while 3 reported turning on a bright light.

Most students in our sample identified as evening types, with $56(70.8 \%)$ identifying as either "strong evening" or "more evening than morning" types. Chronotype was not significantly associated with selfreported sleep duration, time spent in bed, or wake up times on weekdays or weekends. However, evening chronotypes reported significantly later bedtimes during the weekend than participants who identified as morning types $(P=0.025)$.

\section{Sleep habits and aMT6s levels}

Table 2 shows age- and multivariate-adjusted 
Table 2. Age- and multivariate*-adjusted geometric mean aMT6s levels (ng/mg) by selected characteristics

\begin{tabular}{|c|c|c|c|c|}
\hline \multirow[b]{2}{*}{ Characteristic } & \multirow[b]{2}{*}{$\begin{array}{c}\text { Category } \\
\text { Definition }\end{array}$} & \multirow[b]{2}{*}{$\mathbf{N}^{\dagger}$} & \multirow{2}{*}{$\begin{array}{c}\text { Age-adjusted } \\
\text { Geometric mean aMT6s, } \\
95 \% \text { confidence interval }\end{array}$} & \multirow{2}{*}{$\begin{array}{c}\text { Fully adjusted } \\
\text { Geometric mean aMT6s, } \\
\text { 95\% confidence interval }\end{array}$} \\
\hline & & & & \\
\hline \multirow[t]{6}{*}{ Age, years** } & 14 & 20 & $43.3(31.8,60.0)$ & $42.7(30.3,60.3)$ \\
\hline & 15 & 27 & $40.0(30.8-50.9)$ & $44.9(36.5,55.2)$ \\
\hline & 16 & 28 & $35.5(30.0,43.5)$ & $34.3(27.7,43.4)$ \\
\hline & 17 & 17 & $46.0(38.2,55.3)$ & $50.0(41.3,60.6)$ \\
\hline & 18 & 8 & $47.6(31.2,71.5)$ & $46.4(31.4,68.4)$ \\
\hline & $P$ for trend & & 0.743 & 0.809 \\
\hline \multirow[t]{3}{*}{ Gender } & Male & 44 & $37.2(30.2,45.9)$ & $39.3(32.4,47.8)$ \\
\hline & Female & 55 & $43.8(38.7,49.7)$ & $44.7(39.1,51.1)$ \\
\hline & $P$ for difference & & 0.182 & 0.283 \\
\hline \multirow[t]{4}{*}{ BMI, $\mathrm{kg} / \mathrm{m}^{2}$} & $<20$ & 39 & $43.9(36.3,53.0)$ & $43.3(35.4,53.0)$ \\
\hline & $20-25$ & 48 & $38.1(32.1,45.1)$ & $40.5(35.2,46.5)$ \\
\hline & $>25$ & 13 & $43.7(32.7,58.5)$ & $42.7(31.2,58.4)$ \\
\hline & $P$ for trend $d^{\ddagger}$ & & 0.487 & 0.563 \\
\hline \multirow[t]{3}{*}{ Ethnicity } & Caucasian & 67 & $43.0(37.0,50.0)$ & $42.9(37.1,49.7)$ \\
\hline & Non-Caucasian & 32 & $36.9(30.7,44.4)$ & $40.8(34.6,48.1)$ \\
\hline & $P$ for difference & & 0.214 & 0.648 \\
\hline \multirow[t]{7}{*}{ Age at menarche, years ${ }^{\S}$} & 9 & 1 & $39.8(36.8,43.1)$ & - \\
\hline & 10 & 4 & $20.4(12.7,33.0)$ & $18.3(12.2,29.1)$ \\
\hline & 11 & 7 & $32.5(24.1,43.6)$ & $33.5(25.0,44.9)$ \\
\hline & 12 & 16 & $51.3(42.7,61.6)$ & $51.9(43.7,61.4)$ \\
\hline & 13 & 18 & $50.7(42.0,61.2)$ & $50.3(41.6,60.8)$ \\
\hline & 14 & 7 & $50.9(38.7,66.9)$ & $52.9(38.0,73.6)$ \\
\hline & $P$ for trend $d^{\ddagger}$ & & 0.002 & 0.001 \\
\hline \multirow[t]{6}{*}{ Time since menarche, years $* *, \S$} & 1 & 5 & $61.2(36.9,101.4)$ & $58.9(34.6,100.3)$ \\
\hline & 2 & 12 & $55.5(42.0,73.3)$ & $58.1(40.5,83.4)$ \\
\hline & 3 & 11 & $49.6(39.7,61.9)$ & $50.7(41.5,61.9)$ \\
\hline & 4 & 10 & $38.3(30.4,48.2)$ & $39.8(32.7,48.4)$ \\
\hline & $\geq 5$ & 15 & $34.6(26.2,45.7)$ & $32.5(23.2,45.5)$ \\
\hline & $P$ for trend $d^{\ddagger}$ & & 0.017 & 0.024 \\
\hline \multirow[t]{3}{*}{ Oral contraceptive use ${ }^{\S}$} & Yes & 5 & $65.0(46.7,90.5)$ & $65.6(46.7,92.3)$ \\
\hline & No & 50 & $43.1(38.0,49.0)$ & $43.6(38.2,49.7)$ \\
\hline & P for difference & & 0.025 & 0.032 \\
\hline \multirow[t]{3}{*}{ Tobacco use } & Yes & 2 & $27.6(19.3,39.5)$ & $51.7(37.4,71.5)$ \\
\hline & No & 96 & $42.4(37.87,47.5)$ & $42.2(37.5,47.4)$ \\
\hline & $P$ for difference & & 0.026 & 0.266 \\
\hline \multirow[t]{3}{*}{ Alcohol use, past 30 days } & Yes & 18 & $33.4(23.9,46.7)$ & $33.2(24.0,50.3)$ \\
\hline & No & 70 & $44.7(40.2,49.8)$ & $44.9(40.1,50.3)$ \\
\hline & P for difference & & 0.096 & 0.072 \\
\hline \multirow[t]{3}{*}{ Physical activity, hours/week } & $<4$ & 30 & $43.7(35.2,54.2)$ & $44.4(35.1,56.1)$ \\
\hline & $\geq 4$ & 67 & $40.5(35.6,46.1)$ & $41.5(36.5,47.1)$ \\
\hline & P for difference & & 0.545 & 0.614 \\
\hline \multirow[t]{3}{*}{ Use of sleep aids } & Yes & 8 & $48.2(33.3,69.9)$ & $50.4(36.1,70.4)$ \\
\hline & No & 92 & $40.4(35.7,45.6)$ & $41.7(36.9,47.1)$ \\
\hline & P for difference & & 0.366 & 0.290 \\
\hline
\end{tabular}

*Adjusted for: age, gender, ethnicity, BMI, smoking status (yes/no), alcohol consumption (yes/no), physical activity for $4 \mathrm{~h} /$ wk or more (yes/no). ${ }^{\dagger}$ Actual numbers of participants in analyses may vary due to missing covariates; **Not age-adjusted; ${ }^{\ddagger} P$ trend calculated by modeling variables continuously; ${ }^{\circledR}$ Females only. 
geometric mean first morning urinary aMT6s (ng/mg creatinine) levels by participant characteristics. Age was not significantly associated with aMT6s levels in univariate or multivariate analyses $\left(P_{\text {trend }}=0.743\right.$, and 0.809, respectively). Similarly, sex, BMI, race, alcohol use, physical activity, and use of sleep aids showed no significant associations with aMT6s levels, though participants who reported alcohol use had non-significantly lower melatonin than those who did not $(33.2 \mathrm{ng} / \mathrm{mg}$ creatinine v. $44.9 \mathrm{ng} / \mathrm{mg}$ creatinine, $P=0.072$ ). In age-adjusted analyses, older age at menarche was associated with higher aMT6s levels $\left(P_{\text {trend }}=0.002\right)$; multivariate adjustment did not alter this association $\left(P_{\text {trend }}=0.001\right)$. Similarly, time since menarche was also inversely associated with aMT6s levels in both univariate $\left(P_{\text {trend }}=0.017\right)$ and multivariate analyses $\left(P_{\text {trend }}=0.024\right)$. Though based on small numbers of users, oral contraceptive use appeared to be associated with higher aMT6s (43.6 $\mathrm{ng} / \mathrm{mg}$ creatinine v. $65.6 \mathrm{ng} / \mathrm{mg}$ creatinine, $P=0.032$ ).

In multivariate analyses, few self-reported behaviors or light exposures at night significantly predicted circulating aMT6s levels (Table 3). Self-reported exposure to ambient light at night in the bedroom did not appear to significantly affect nightly melatonin

Table 3. Multivariate-adjusted* geometric mean aMT6s (ng/mg creatinine), by category of light at night and sleep duration among 100 study participants

\begin{tabular}{|c|c|c|c|}
\hline Characteristic & Category Definition & $\mathbf{N}^{\dagger}$ & Geometric mean aMT6s, $95 \%$ confidence interval \\
\hline \multirow{3}{*}{$\begin{array}{l}\text { Light outside bedroom } \\
\text { window }\end{array}$} & Yes & 45 & $45.8(39.1,53.7)$ \\
\hline & No & 54 & $39.6(33.4,46.9)$ \\
\hline & P for difference & & 0.228 \\
\hline \multirow{4}{*}{$\begin{array}{l}\text { Electronic use after } \\
\text { lights out, weekdays }\end{array}$} & $<1$ hour & 79 & $43.9(38.69,49.7)$ \\
\hline & $1-2$ hours & 18 & $34.7(27.3,44.1)$ \\
\hline & $\geq 3$ hours & 3 & $55.3(43.1,70.8)$ \\
\hline & $P$ for trend $d^{\ddagger}$ & & 0.611 \\
\hline \multirow{4}{*}{$\begin{array}{l}\text { Electronic use after } \\
\text { lights out, weekends }\end{array}$} & $<1$ hour & 51 & $45.6(39.2,53.0)$ \\
\hline & $1-2$ hours & 41 & $39.7(32.8,48.1)$ \\
\hline & $\geq 3$ hours & 8 & $36.8(27.0,50.1)$ \\
\hline & $P$ for trend $d^{\ddagger}$ & & 0.163 \\
\hline \multirow[t]{4}{*}{ Sleep interrupted } & Never interrupted & 73 & $43.0(37.8,48.9)$ \\
\hline & 1-4 times/night without light on & 21 & $43.1(33.5,55.6)$ \\
\hline & 1-4 times/night with light on & 4 & $24.6(14.9,40.5)$ \\
\hline & $\begin{array}{l}\text { P for difference (never vs. 1-4 times/ } \\
\text { night with light on) }\end{array}$ & & $0.032 *$ \\
\hline \multirow{3}{*}{$\begin{array}{l}\text { Light or TV on in or near } \\
\text { bedroom overnight }\end{array}$} & Yes & 22 & $43.7(37.4,51.1)$ \\
\hline & No & 78 & $41.9(36.5,48.1)$ \\
\hline & P for difference & & 0.647 \\
\hline \multirow{5}{*}{$\begin{array}{l}\text { Average self-reported } \\
\text { sleep duration, } \\
\text { weekdays }\end{array}$} & $<5$ hours & 3 & $73.4(60.3,89.3)$ \\
\hline & 5-6 hours & 28 & $35.5(27.6,45.5)$ \\
\hline & $7-8$ hours & 66 & $44.1(38.4,50.7)$ \\
\hline & $\geq 9$ hours & 2 & $30.8(16.0,59.5)$ \\
\hline & $P$ for trend & & 0.691 \\
\hline
\end{tabular}

*Adjusted for: age, gender, ethnicity, BMI, alcohol consumption (yes/no), physical activity for 4 h/wk or more (yes/no). ${ }^{\dagger}$ Actual numbers of participants in analyses may vary due to missing covariates; ${ }^{\star} P$ trend calculated by modeling median of each category as continuous, or by modeling ordinal categories continuously. 
Table 3. (continued from previous page) Multivariate-adjusted* geometric mean aMT6s (ng/mg creatinine), by category of light at night and sleep duration among 100 study participants

\begin{tabular}{|c|c|c|c|}
\hline Characteristic & Category Definition & $\mathbf{N}^{\dagger}$ & Geometric mean aMT6s, $95 \%$ confidence interval \\
\hline \multirow{6}{*}{$\begin{array}{l}\text { Average self-reported } \\
\text { sleep duration, } \\
\text { weekends }\end{array}$} & $<5$ hours & 3 & $40.9(25.2,66.6)$ \\
\hline & $5-6$ hours & 7 & $23.4(12.7,43.1)$ \\
\hline & $7-8$ hours & 28 & $44.3(36.4,53.9)$ \\
\hline & $9-10$ hours & 49 & $45.2(39.1,52.2)$ \\
\hline & $>10$ hours & 12 & $41.7(31.5,55.2)$ \\
\hline & P for trend & & 0.121 \\
\hline \multirow{7}{*}{$\begin{array}{l}\text { Self-reported brightness } \\
\text { level in bedroom, } \\
\text { weekdays }\end{array}$} & Level 1 (wearing a mask) & 0 & - \\
\hline & Level 2 (cannot see hands) & 32 & $39.7(31.5,50.1)$ \\
\hline & Level 3 (can see to end of bed) & 24 & $40.8(33.4,49.8)$ \\
\hline & Level 4 (can see across room) & 38 & $44.5(36.8,53.7)$ \\
\hline & Level 5 (can barely read) & 3 & $103.9(68.8,156.8)$ \\
\hline & Level 6 (can read comfortably) & 3 & $31.3(19.8,49.7)$ \\
\hline & P for trend & & 0.403 \\
\hline \multirow{7}{*}{$\begin{array}{l}\text { Self-reported brightness } \\
\text { level in bedroom, night } \\
\text { before collection }\end{array}$} & Level 1 (wearing a mask) & 0 & - \\
\hline & Level 2 (cannot see hands) & 30 & $39.0(30.5,49.9)$ \\
\hline & Level 3 (can see to end of bed) & 21 & $40.0(32.0,50.1)$ \\
\hline & Level 4 (can see across room) & 33 & $46.8(39.3,55.8)$ \\
\hline & Level 5 (can barely read) & 3 & $73.5(41.4,130.5)$ \\
\hline & Level 6 (can read comfortably) & 2 & $32.4(26.1,40.2)$ \\
\hline & Pfor trend & & 0.096 \\
\hline \multirow{4}{*}{$\begin{array}{l}\text { Average reported } \\
\text { bedtime, weekdays }\end{array}$} & Before $11 \mathrm{pm}$ & 56 & $44.6(38.0,52.3)$ \\
\hline & $11 \mathrm{pm}$ to midnight & 38 & $38.4(31.8,46.2)$ \\
\hline & After midnight & 4 & $41.8(25.8,67.7)$ \\
\hline & $P$ for trend & & 0.317 \\
\hline \multirow{4}{*}{$\begin{array}{l}\text { Average reported } \\
\text { bedtime, weekends }\end{array}$} & Before $11 \mathrm{pm}$ & 12 & $52.5(42.4,64.9)$ \\
\hline & $11 \mathrm{pm}$ to midnight & 24 & $48.8(40.8,58.4)$ \\
\hline & After midnight & 61 & $38.0(32.4,44.5)$ \\
\hline & P for trend & & 0.007 \\
\hline \multirow[t]{5}{*}{ Chronotype } & Definite morning type & 5 & $50.7(40.5,63.4)$ \\
\hline & More morning type than evening & 18 & $32.4(23.6,44.4)$ \\
\hline & More evening type than morning & 39 & $45.2(38.8,52.8)$ \\
\hline & Definite evening type & 17 & $50.2(38.7,65.1)$ \\
\hline & P for trend & & 0.157 \\
\hline
\end{tabular}

*Adjusted for: age, gender, ethnicity, BMI, alcohol consumption (yes/no), physical activity for $4 \mathrm{~h} /$ wk or more (yes/no). ${ }^{\dagger}$ Actual numbers of participants in analyses may vary due to missing covariates; ${ }^{\ddagger} P$ trend calculated by modeling median of each category as continuous, or by modeling ordinal categories continuously.

levels. AMT6s levels did not meaningfully vary by self-reported exposure to light at night outside the bedroom window, electronic use after lights out, whether or not light or a TV was on during sleep, or brightness levels in the bedroom at night on weekdays, weekends, or the night before the collection. 
No significant trend between aMT6s levels and reported bedtime on the weeknights was observed $\left(P_{\text {trend }}=0.317\right)$, though later reported bedtimes on the weekend were significantly inversely associated with aMT6s levels $(38.0 \mathrm{ng} / \mathrm{mg}$ creatinine for bedtimes after midnight v. $52.5 \mathrm{ng} / \mathrm{mg}$ creatinine for bedtimes before $\left.11 \mathrm{pm}, P_{\text {trend }}=0.007\right)$. Lastly, neither self-reported sleep duration nor time spent in bed on either weekdays or weekends appeared to be a significant predictor of aMT6 levels.

We also explored associations between aMT6s levels and self-reported light exposure during sleep interruption. Borderline significantly lower aMT6s levels were found among the four participants who turned the light on if their sleep got interrupted at night (24.6 ng/mg creatinine, 95\% CI: 14.9, 40.5) when compared to adolescents with interrupted sleep who did not turn the light on $(43.1 \mathrm{ng} / \mathrm{mg}$ creatinine, $95 \%$ CI: 33.5, 55.6, $\left.P_{\text {difference }}=0.050\right)$ and significantly lower levels were observed when compared to those who had their sleep never interrupted $(43.0 \mathrm{ng} / \mathrm{mg}$ creatinine, $95 \%$ CI: $\left.37.8,48.9, P_{\text {difference }}=0.032\right)$. No significant differences were observed comparing participants with interrupted sleep who did not turn the light on compared to those whose sleep was not interrupted $\left(P_{\text {difference }}=0.981\right)$.

Self-reported chronotype was not associated with aMT6s levels $\left(P_{\text {trend }}=0.157\right)$, though there was some suggestion of a non-linear relationship. aMT6s levels were highest among those who self-identified as "definite" morning or evening types, and lower among those who reported being "more" of a morning or evening type.

\section{DISCUSSION}

This study is among the first to examine relationships between adolescent sleep patterns and behaviors and urinary measures of melatonin. In our sample of high school students, we found that few aspects of adolescent sleep behavior and self-reported nocturnal light exposure were associated with urinary aMT6s levels.

Though exposure to light at night is known to suppress melatonin secretion, no associations between any self-reported measure of ambient light in or around the bedroom and aMT6s levels were observed in the present study. While laboratory studies have demonstrated the ability of light at night to suppress melatonin levels, the intensity of residential light may be orders of magnitude less intense than light used in experimental studies and insufficient to quantifiably affect melatonin secretion. ${ }^{16}$ The effect of ambient light on melatonin is likely further reduced during sleep, when eyelids are closed, as mean light transmittance across the human eyelid has been reported to be as low as $1 \% .{ }^{17}$ While high-intensity light exposure delivered across the eyelids by an over-eye mask has been shown to suppress serum melatonin levels and delay dim light melatonin onset (DLMO) in young adults, ${ }^{18}$ these experimental conditions are not representative of the light exposures experienced by our study participants.

Use of electronic devices after turning off the lights was not found to be a significant predictor of aMT6s levels. Melatonin is suppressed by specific wavelengths of blue light, especially in the 46-477 $\mathrm{nm}$ range $;{ }^{19}$ many electronic devices also emit light in this range of the spectrum. Evidence in young adults suggests that devices with light emitting diode (LED) displays are stronger suppressors of melatonin than those that emit white light ${ }^{20,21}$ and that significant suppression may occur only after long durations of use. ${ }^{22}$ In one small experimental study, nighttime exposure to light from a computer monitor was not associated with significantly decreased levels of salivary melatonin in college students. ${ }^{23}$ Our results are significant given the prevalence of nighttime electronic use among adolescents; in a recent national survey, the proportions of 13-18 year old respondents who reported regularly using a cellphone or a computer in the hour before trying to go to sleep were $72 \%$ and $60 \%$, respectively. ${ }^{24}$

Interestingly, participants in our study who reported the latest weekend bedtimes were found to have the lowest midweek melatonin levels. Compared to adults, adolescents are likely to have a delayed circadian phase, even in the absence of social pressures $^{2,25,26}$ and these phase delays may manifest as a later DLMO. ${ }^{2,27}$ We hypothesize that falling asleep later on the weekends may be an indicator of delayed circadian phase. Because all students in our study began school at the same time $(7: 45 \mathrm{am})$ on the day of urine 
collection, it is reasonable to assume that participants had a similar wake-up time. Among students with a delayed circadian phase, the time from DLMO to this fixed wake-up time was shorter, and as such, their first morning urine sample reflected an incomplete overnight secretion of melatonin. Therefore, low melatonin levels may be identifying a group of students whose circadian tendencies are most in conflict with school start times. However, the lack of an association between weekend bedtimes and self-reported chronotype makes this finding difficult to interpret.

Our suggestive finding that exposure to light during sleep interruption is associated with lower aMT6s levels is given some support by evidence demonstrating that exposure to light at night suppresses the secretion of melatonin by the pineal gland. ${ }^{9,28,29}$ However, experimental study protocols generally involve continuous exposure to light for several hours during normal sleep hours at relatively high intensities of light. ${ }^{9,10}$ In contrast, only one participant in our study reported leaving lights on for 10 or more minutes during sleep interruption. Given that light interruption in our study was brief and that the primary reason for sleep interruption may have been to urinate, which could have lowered first-morning urinary melatonin measures, these results must be interpreted with caution. Further, the small number of participants who reported exposure to light during sleep interruption must be considered.

Though aMT6s levels and BMI have been found to be correlated in adults, ${ }^{11}$ we could not confirm this association in our adolescent population, which is consistent with a recent study in adolescent Korean girls. ${ }^{30}$ Additionally, we observed a non-significant decrease in aMT6s levels among participants who reported alcohol use: alcohol consumption has been correlated with decreased melatonin levels in some, ${ }^{31,32}$ though not all, ${ }^{11,33}$ studies of adults. In multivariate analyses, higher melatonin levels were observed among girls who reported a later age at menarche. While age at menarche was not associated with aMT6s levels in adult women, ${ }^{11}$ Crowley et al ${ }^{14}$ recently reported that advanced Tanner stage was associated with decreases in overnight melatonin secretion among adolescents. Since menarche can be used as a pubertal marker, ${ }^{35}$ our results appear consistent with these prior findings, as girls in our study who experienced menarche earlier may have been more pubertally advanced than girls with more recent menarche. Our finding that time since menarche was inversely associated with aMT6s levels also supports this hypothesis. Lastly, in multivariate analyses, we found that girls had nonsignificantly higher aMT6s levels compared to boys. Higher melatonin secretion in adolescent girls was reported by Crowley et al, ${ }^{34}$ though other studies of melatonin in adolescents did not find significant sex differences. ${ }^{36-38}$

This study has several strengths. First, our sample size is larger than most other studies of melatonin in adolescents and is the first to observe lifestyle correlates of urinary aMT6s levels in an adolescent population. Previous work has shown that single measurements of aMT6s in first-morning urine are highly correlated to peak and total plasma melatonin levels ${ }^{37}$ and that aMT6s levels from samples collected in the field and the laboratory were in close agreement. ${ }^{39}$ Therefore, our measurements of aMT6s in first-morning urine provide an adequate representation of nocturnal melatonin levels, as well as a non-intrusive method of sample collection that is acceptable to adolescent participants.

The main limitation of our study is its crosssectional nature. In adults, levels of urinary aMT6s have been shown to have high reproducibility over long periods, ${ }^{40}$ though it remains unclear whether a single measurement of melatonin in adolescents is an adequate representation of long-term levels. Some evidence suggests that melatonin levels decline throughout childhood and puberty, ${ }^{34,41,42}$ though other evidence suggests that urinary aMT6s secretion may remain constant after the onset of puberty. ${ }^{36}$ Griefahn et $\mathrm{al}^{37}$ reported that despite huge between-subject variability, within-subject levels of urinary excretion of melatonin metabolite remained constant from ages 3 to 18 . An additional limitation of our study was that it relied on self-reported light levels and not objective, standardized measures of ambient light. However, these light levels are more indicative of the levels that adolescents are habitually exposed to, in contrast to the light stimuli used in many experimental protocols.

This is the first study to date to present correlations between sleep patterns and behaviors and urinary melatonin levels in an adolescent population. Our results 
suggest that the nighttime behaviors of adolescents by and large do not impact urinary melatonin levels. Given the prevalence of sleep deprivation among adolescents and the gravity of its consequences, more research is needed to elucidate the pathways through which such sleep-related factors may impact physiology.

\section{Human Subjects Approval Statement}

Written consent was obtained from all participants and from the parent or guardian of each participant. All procedures were conducted with approval of the Ossining High School Institutional Review Board.

\section{ACKNOWLEDGEMENTS}

Funding provided by NIH/NCI (T32 CA0090001, R25 CA098566) and R01OH009803 (PI: Schernhammer).

\section{REFERENCES}

1. National Sleep Foundation, 2006 Teens and Sleep. http://www.sleepfoundation.org/article/sleep-americapolls/2006-teens-and-sleep

2. Crowley SJ, Acebo C, Fallone G, Carskadon MA, 2006 Estimating dim light melatonin onset (DLMO) phase in adolescents using summer or school-year sleep/wake schedules. Sleep 29: 1632-1641.

3. Hagenauer MH, Perryman JI, Lee TM, Carskadon MA, 2009 Adolescent changes in the homeostatic and circadian regulation of sleep. Dev Neurosci 31: 276284.

4. Smaldone A, Honig JC, Byrne MW, 2007 Sleepless in America: inadequate sleep and relationships to health and well-being of our nation's children. Pediatrics 119: Suppl 1: 29-37.

5. Wolfson AR, Carskadon MA, 1998 Sleep schedules and daytime functioning in adolescents. Child Dev 69: 875-887.

6. Chen X, Beydoun MA, Wang Y, 2008 Is sleep duration associated with childhood obesity? A systematic review and meta-analysis. Obesity (Silver Spring) 16: 265-274.

7. Schernhammer ES, Berrino F, Krogh V, et al, 2008 Urinary 6-sulfatoxymelatonin levels and risk of breast cancer in postmenopausal women. J Natl Cancer Inst 100: 898-905.

8. Owens JA, Belon K, Moss P, 2010 Impact of delaying school start time on adolescent sleep, mood, and behavior. Arch Pediatr Adolesc Med 164: 608-614.

9. Zeitzer JM, Dijk DJ, Kronauer R, Brown E, Czeisler C, 2000 Sensitivity of the human circadian pacemaker to nocturnal light: melatonin phase resetting and sup- pression. J Physiol (Lond) 526 Pt 3: 695-702.

10. Gooley JJ, Chamberlain K, Smith KA, et al, 2011 Exposure to room light before bedtime suppresses melatonin onset and shortens melatonin duration in humans. J Clin Endocrinol Metab 96: E463-472.

11. Schernhammer ES, Kroenke CH, Dowsett M, Folkerd E, Hankinson SE, 2006 Urinary 6-sulfatoxymelatonin levels and their correlations with lifestyle factors and steroid hormone levels. J Pineal Res 40: 116-124.

12. Davis S, Mirick DK, Stevens RG, 2001 Night shift work, light at night, and risk of breast cancer. J Natl Cancer Inst 93: 1557-1562.

13. Horne JA, Ostberg OA, 1976 Self-assessment questionnaire to determine morningness-eveningness in human circadian rhythms. Int J Chronobiol 4: 97-110.

14. Medgdal SP, Schernhammer ES, 2007 Correlates for poor sleepers in a Los Angeles high school. Sleep Med 9: 60-63.

15. Rosner B, 1983 Percentage Points for a Generalized ESD Many-Outlier Procedure. Technometrics 25: 165172.

16. Rea MS, Figueiro MG, Bierman A, Bullough JD, 2010 Circadian light. J Circadian Rhythms 8: 2.

17. Bierman A, Figueiro MG, Rea MS, 2011 Measuring and predicting eyelid spectral transmittance. J Biomed Opt 16: 067011.

18. Figueiro MG, Rea MS, 2012 Preliminary evidence that light through the eyelids can suppress melatonin and phase shift dim light melatonin onset. BMC Res Notes 5: 221.

19. Brainard GC, Sliney D, Hanifin JP, et al, 2008 Sensitivity of the human circadian system to short-wavelength (420-nm) light. J Biol Rhythms 23: 379-386.

20. Cajochen C, Frey S, Anders D, et al, 2011 Evening exposure to a light-emitting diodes (LED)-backlit computer screen affects circadian physiology and cognitive performance. J Appl Physiol 110: 1432-1438.

21. West KE, Jablonski MR, Warfield B, et al, 2011 Blue light from light-emitting diodes elicits a dose-dependent suppression of melatonin in humans. J Appl Physiol 110: 619-626.

22. Wood B, Rea MS, Plitnick B, Figueiro MG, 2013 Light level and duration of exposure determine the impact of self-luminous tablets on melatonin suppression. Appl Ergon 44: 237-240.

23. Figueiro MG, Wood B, Plitnick B, Rea MS, 2011 The impact of light from computer monitors on melatonin levels in college students. Neuro Endocrinol Lett 32: 158-163.

24. National Sleep Foundation, 2011 Sleep in America Poll: Communications Technology in the Bedroom. http://www.sleepfoundation.org/article/sleep-americapolls/2011-communications-technology-use-and-sleep

25. Carskadon MA, Vieira C, Acebo C, 1993 Association between puberty and delayed phase preference. Sleep 16: $258-262$. 
26. Carskadon MA, Acebo C, Jenni OG, 2004 Regulation of adolescent sleep: implications for behavior. Ann NY Acad Sci 1021: 276-291.

27. Burgess HJ, Eastman CI, 2005 The dim light melatonin onset following fixed and free sleep schedules. J Sleep Res 14: 229-237.

28. Bojkowski CJ, Arendt J, 1990 Factors influencing urinary 6-sulphatoxymelatonin, a major melatonin metabolite, in normal human subjects. Clin Endocrinol (Oxf) 33: 435-444.

29. Lewy AJ, Wehr TA, Goodwin FK, Newsome DA, Markey SP, 1980 Light suppresses melatonin secretion in humans. Science 210: 1267-1269.

30. Lee J, Yoon J, Lee JA, Lee SY, Shin CH, Yang SW, 2012 Urinary 6-sulfatoxymelatonin level in girls and its relationship with obesity. Korean J Pediatr 55: 344349.

31. Rupp TL, Acebo C, Carskadon MA, 2007 Evening alcohol suppresses salivary melatonin in young adults. Chronobiol Int 24: 463-470.

32. Stevens RG, Davis S, Mirick DK, Kheifets L, Kaune W, 2000 Alcohol consumption and urinary concentration of 6-sulfatoxymelatonin in healthy women. Epidemiology 11: 660-665.

33. Hartman TJ, Mahabir S, Baer DJ, et al, 2012 Moderate alcohol consumption and 24-hour urinary levels of melatonin in postmenopausal women. J Clin Endocrinol Metabol 97: E65-E68.

34. Crowley SJ, Acebo C, Carskadon MA, 2012 Human puberty: Salivary melatonin profiles in constant condi- tions. Dev Psychobiol 54: 468-473.

35. Frey S, Balu S, Greusing S, Rothen N, Cajochen C, 2009 Consequences of the timing of menarche on female adolescent sleep phase preference. PLoS ONE 4: e5217.

36. Cavallo A, Dolan LM, 1996 6-Hydroxymelatonin sulfate excretion in human puberty. J Pineal Res 21: 225-230.

37. Griefahn B, Bröde P, Blaszkewicz M, Remer T, 2003 Melatonin production during childhood and adolescence: a longitudinal study on the excretion of urinary 6-hydroxymelatonin sulfate. J Pineal Res 34: 26-31.

38. Salti R, Galluzzi F, Bindi G, et al, 2000 Nocturnal melatonin patterns in children. J Clin Endocrinol Metab 85: 2137-2144.

39. Cook MR, Graham C, Kavet R, Stevens RG, Davis S, Kheifets L, 2000 Morning urinary assessment of nocturnal melatonin secretion in older women. J Pineal Res 28: 41-47.

40. Schernhammer ES, Rosner B, Willett WC, Laden F, Colditz GA, Hankinson SE, 2004 Epidemiology of urinary melatonin in women and its relation to other hormones and night work. Cancer Epidemiol Biomarkers Prev 13: 936-943.

41. Waldhauser F, Steger H, 1986 Changes in melatonin secretion with age and pubescence. J Neural Transm Suppl. 21: 183-197.

42. Waldhauser F, Weiszenbacher G, Frisch H, Zeitlhuber U, Waldhauser M, Wurtman RJ, 1984 Fall in nocturnal serum melatonin during prepuberty and pubescence. Lancet 1: 362-365. 